Revista Mexicana de Astronomía y Astrofísica, 55, 321-335 (2019)

DOI: https://doi.org/10.22201/ia.01851101p.2019.55.02.17

\title{
CHAOS IN GROWING BAR MODELS
}

\author{
Lucas Antonio Caritá ${ }^{1,2,3}$, Irapuan Rodrigues ${ }^{2}$, Ivânio Puerari ${ }^{3}$, and Luiz Eduardo Camargo Aranha \\ Schiavo $^{2,4}$ \\ Received December 30 2018; accepted August 32019
}

\begin{abstract}
This paper aims to verify the influence of the bar, its pattern speed $\left(\Omega_{b}\right)$ and its rate of growth on the stability of the orbits in gravitational potentials. We studied the nature of the orbits in potentials representing galaxies with growing bars, where a linear growth was assumed. In order to study the stability of the orbits we applied SALI. We studied six models in which the bar dimensions were fixed, but we varied their pattern speed and time of bar growth. We found that when the bar growth is faster, more chaos is generated and we also noted that the higher the $\Omega_{b}$, the greater its influence on the system dynamics. The initial positions of the orbits that became chaotic were located in a well-defined ring-like region, confined between the ILR and CR resonances. There was also an indication that the retrograde orbits, although much scarcer, are more conductive to chaos when they do exist.
\end{abstract}

\section{RESUMEN}

Este trabajo verifica la influencia de la barra, de su velocidad angular $\left(\Omega_{b}\right)$ y de su tasa de crecimiento en la estabilidad de las órbitas en potenciales gravitacionales. Estudiamos órbitas en potenciales representando galaxias con barras en crecimiento, asumiendo un crecimiento lineal. Para estudiar la estabilidad de las órbitas aplicamos SALI. Estudiamos seis modelos con dimensiones fijas de la barra, pero variamos la velocidad y el tiempo de crecimiento de la misma. Evidenciamos que cuando el crecimiento de la barra es más rápido, se genera más caos y también observamos que cuanto mayor es $\Omega_{b}$, mayor será su influencia en la dinámica del sistema. Las posiciones iniciales de las órbitas que se han vuelto caóticas quedan ubicadas en una región anular bien definida, confinada entre ILR y CR. Las órbitas retrógradas, aunque mucho más escasas, parecen ser más propicias al el caos.

Key Words: chaos — galaxies: general — galaxies: kinematics and dynamics galaxies: spiral

\section{INTRODUCTION}

Approximately $65 \%$ of disk galaxies show bar-like structures (Eskridge et al. 2000; Sheth et al. 2003). The characteristic of their bars varies considerably, from faint weak bars to prominent, strong and massive bars. By computational integration of stellar orbits in gravitational potential models, it is possible to study the dynamics and stability of this type of

\footnotetext{
${ }^{1}$ Instituto Federal de Educação, Ciência e Tecnologia de São Paulo (IFSP), São José dos Campos, Brasil.

${ }^{2}$ Universidade do Vale do Paraíba (UNIVAP), São José dos Campos, Brasil.

${ }^{3}$ Instituto Nacional de Astrofísica, Óptica y Electrónica (INAOE), Puebla, México.

${ }^{4}$ Universidade Estadual de Campinas (UNICAMP), Campinas, Brasil.
}

galaxy. Indeed, stellar orbits supported by a galactic potential are the basic constituents of any galactic structure. Understanding the behavior of stellar orbits is essential for understanding the formation and evolution of these structures.

In recent works, integrations of orbits in fixedparameter bar potentials have been performed; it was concluded that for sufficiently large bar axial ratios, stable orbits having propeller shapes have a great influence on bar structure (Kaufmann \& Patsis 2005). Several types of resonant orbits can shape the bar structure, besides the $x_{1}$ orbital family. Although the $x_{1}$ family is considered to be the backbone of $2 \mathrm{D}$ bars, in the case of $3 \mathrm{D}$ this family is aided by a tree of its 3D bifurcating families (Skokos 
et al. 2002b). All other bar-supporting orbits are candidates for supporting the inner parts of the bar (Gajda et al. 2016; Patsis \& Katsanikas 2014). In recent studies it was verified that more massive bars have a greater tendency for chaotic orbits to occur, whereas weaker bars are less affected by chaos (Manos \& Athanassoula 2011; Caritá et al. 2017).

However, it is agreed that the formation of a bar is a long and complex secular process, which may have several histories. It is also agreed that no galaxy is born barred: the bar can form, change (increase, decrease, rotate etc.) and extinguish itself with time, in processes that depend on the parameters of the galaxies that host them (Bournaud \& Combes 2002). Regarding this trend, Manos \& Machado (2014), Machado \& Manos (2016) and Chaves-Velasquez et al. (2017) presented studies on the regular or chaotic character of orbits in time-dependent barred galaxy potentials based on an N-body simulation. They extracted parameters of bar evolution from the simulation for certain times, treating each snapshot as a time-independent model.

In Caritá et al. (2017), the SALI (Smaller Alignment Index) method was applied (Skokos 2001), to study the stability of stellar orbits in the gravitational potential of barred galaxies with fixed parameters, in which the theoretical models based on Manos \& Athanassoula (2011) were used. In that work, we were exclusively interested in evaluating the influence of the bar parameters on the occurrence of chaos in the stellar orbits.

In the present paper we propose a new approach by adding some new ingredients. First of all, we study six models based on observational properties of the grand design barred galaxy NGC 936, from which we borrow the main parameters, as presented in detail in Appendix A. We also introduce analytically the growth of the bar, i.e., we set time-dependent evolving bar potentials. Moreover, we verify the influence of the pattern speed and the rate of growth of the bar on the stability of the orbits.

To perform the orbital integrations and SALI calculation, we used a slight adaptation of the LPVIcode program (Carpintero et al. 2014), which is a fully operational code, implemented in Fortran 77, that efficiently calculates 10 different chaos indicators for dynamic systems, regardless of the number of dimensions, SALI being one of them.

\section{METHODOLOGY}

\subsection{The Smaller Alignment Index (SALI)}

In order to define SALI, let us consider a Hamiltonian flow of $N$ degrees of freedom, an orbit in the $2 N$ dimensional phase space with initial condition $x(0)=\left(x_{1}(0), \cdots, x_{2 N}(0)\right)$ and two normalized deviation vectors $\widehat{\mathbf{w}_{1}}(0), \widehat{\mathbf{w}_{2}}(0)$ from the initial condition $x(0)$.

We define

$$
S A L I(t):=\min \left\{\left\|\widehat{\mathbf{w}}_{1}(t)-\widehat{\mathbf{w}}_{2}(t)\right\|,\left\|\widehat{\mathbf{w}}_{1}(t)+\widehat{\mathbf{w}}_{2}(t)\right\|\right\},
$$

where the quantities $\left\|\widehat{\mathbf{w}}_{1}(t)-\widehat{\mathbf{w}}_{2}(t)\right\|$ and $\| \widehat{\mathbf{w}}_{1}(t)+$ $\widehat{\mathbf{w}}_{2}(t) \|$ are called Parallel Alignment Index and Antiparallel Alignment Index, respectively.

It is evident that $S A L I(t) \in[0, \sqrt{2}]$ and when $S A L I=0$ the two normalized vectors have the same direction, being equal or opposite.

The SALI value is a very useful tool for detecting chaos in Hamiltonian systems. Chaotic or regular motions are easily distinguishable applying the SALI method. In the case of chaotic orbits, the deviation vectors $\widehat{\mathbf{w}}_{1}(t)$ and $\widehat{\mathbf{w}}_{2}(t)$ align in the direction defined by the Maximum Lyapunov Exponent (MLE) and $S A L I(t)$ falls exponentially to zero:

$$
S A L I(t) \propto e^{-\left(L_{1}-L_{2}\right) t},
$$

with $L_{1}$ and $L_{2}$ the two largest Lyapunov exponents.

Furthermore, for regular motions the orbits develop on a phase space torus and eventually the vectors $\widehat{\mathbf{w}}_{1}(t)$ and $\widehat{\mathbf{w}}_{2}(t)$ fall in the torus tangent space, following a $t^{-1}$ time dependence. In this case, SALI oscillates at nonzero values:

$$
S A L I(t) \approx \text { constant }>0 .
$$

We have a clear distinction between ordered and chaotic behaviors using the SALI method in Hamiltonian systems. For mathematical SALI details, we recommend reading the papers Skokos (2001); Skokos et al. (2002a, 2003, 2004).

\subsection{Mathematical Modeling of the Gravitational Potential}

In this investigation, we used the gravitational potential divided into three basic components: bulge, disk and bar, according to the following equation:

$$
\Phi_{\text {Total }}=\Phi_{\text {Bulge }}+\Phi_{\text {Disk }}+\Phi_{\text {Bar }} .
$$

Each component of equation (4) was mathematically modeled according to a classical potential: the Plummer potential was used for the bulge (Plummer 1911), Miyamoto-Nagai's for the disk (Miyamoto \& Nagai 1975) and Ferrers' for the bar (Ferrers 1877). This way of representing the total gravitational potential has been extensively used in many articles, 
such as Patsis (2002); Manos \& Athanassoula (2011); Skokos et al. (2002c,d); Patsis et al. $(2002,2003)$ and Caritá et al. (2017).

The Plummer potential is written as:

$$
\Phi_{\text {Bulge }}=-\frac{G M_{S}}{\sqrt{x^{2}+y^{2}+z^{2}+\epsilon^{2}}},
$$

where $\epsilon$ is the scale-length of the bulge, $M_{S}$ is its total mass, and $G$ is the gravitational constant.

The Miyamoto-Nagai's potential is written as:

$$
\Phi_{\text {Disk }}=-\frac{G M_{D}}{\sqrt{x^{2}+y^{2}+\left(A+\sqrt{z^{2}+B^{2}}\right)^{2}}},
$$

where $M_{D}$ is the total disk mass, $A$ and $B$ are its horizontal and vertical scale-lengths, and $G$ is the gravitational constant.

The Ferrers' potential is written as:

$$
\Phi_{B a r}=-\pi G a b c \frac{\rho_{c}}{3} \int_{\lambda}^{\infty} \frac{d u}{\Delta(u)}\left(1-m^{2}(u)\right)^{3},
$$

where $m^{2}(u)=\frac{x^{2}}{a^{2}+u}+\frac{y^{2}}{b^{2}+u}+\frac{z^{2}}{c^{2}+u}, \quad \Delta^{2}(u)=$ $\left(a^{2}+u\right)\left(b^{2}+u\right)\left(c^{2}+u\right), \lambda$ is the positive solution of $m^{2}(\lambda)=1$ for the region outside the bar $(m \geq 1)$ and $\lambda=0$ for the region inside the bar $(m<1)$.

In this last potential, the density is given by

$$
\rho_{B}(x, y, z)=\left\{\begin{array}{l}
\rho_{c}\left(1-m^{2}\right)^{2}, \quad m<1 \\
0, \quad m \geq 1
\end{array}\right.
$$

where the central density is $\rho_{c}=\frac{105}{32 \pi} \frac{G M_{B}}{a b c}, M_{B}$ is the bar mass and $m^{2}=\frac{x^{2}}{a^{2}}+\frac{y^{2}}{b^{2}}+\frac{z^{2}}{c^{2}}$, where $a>b>c>0$ are the semi-axes of the ellipsoid which represents the bar.

In order to implement this bar model computationally, we used the analytical version given by Dr. Pfenniger, who kindly provided us with his Fortran 77 routine of the Ferrers potential. In this routine, the polynomial form of the Ferrers potential (Pfenniger 1984; Caritá et al. 2017) was used.

In the course of this work, the SALI method was applied to study stellar orbits in a gravitational potential of barred galaxies, since the motion of a test particle in a rotating 3-dimensional model of a barred galaxy is given by the Hamiltonian:

$$
\begin{aligned}
& H\left(x, y, z, p_{x}, p_{y}, p_{z}\right)=\frac{1}{2}\left(p_{x}^{2}+p_{y}^{2}+p_{z}^{2}\right)+ \\
& \Phi_{\text {Total }}(x, y, z)-\Omega_{b}\left(x p_{y}-y p_{x}\right)
\end{aligned}
$$

where the bar rotates around the $z$-axis; $x$ and $y$ respectively are the major and minor galactic bar axes, $\Phi_{\text {Total }}$ is the total gravitational potential given by equation (4) and $\Omega_{b}$ is the bar pattern speed.

We emphasize that in order to follow the evolution of the orbits and that of their deviation vectors (for SALI computation), it is necessary to know the equations of motion and the variational equations linked to the Hamiltonian (9). The corresponding motion and variational equations can be checked in Manos \& Machado (2014).

To study orbit stability in models with 2 degrees of freedom, in our calculations, $z=0$ and $p_{z}=0$ were adopted in the Hamiltonian shown in equation (9).

\subsection{Implementation and Computation Using the LP-VIcode}

To perform the orbital integrations and SALI calculation, the LP-VIcode (Carpintero et al. 2014) was employed, which is freely available at http: //lp-vicode.fcaglp.unlp.edu.ar/.

LP-VIcode is an operational code in Fortran 77 that efficiently calculates 10 chaos indicators for dynamic systems, including SALI. The program reads the initial conditions for one or more orbits, integrates them (using a Bulirsch-Stoer integrator), and calculates the equations of the chosen chaos indicators. More details about the structure and operation of the LP-VIcode can be found in Carpintero et al. (2013) and Carpintero et al. (2014).

In order to integrate orbits using the program and to study their stability, the user must provide the potential expressions and the motion and variational equations. That is, there is an external routine where these equations must be written in Fortran 77 by the user.

Two actions performed in the LP-VIcode implementation and adaptation stage should be highlighted in this section: the first is the adjustments that were made in order to implement a rotating coordinate system in the code (since the general equations (of motion and variational) present in the original main program consider only a static reference frame; it is known that in order to model a rotating galactic bar it is necessary to consider a coordinate system that rotates along with the bar. To do this we inserted the pattern speed $\Omega_{b}$ in the motion and variational equations in the LP-VIcode main program transforming the original equations into the form which Manos \& Athanassoula (2011) displayed in their text. The second important action was bar growth. As previously explained, in this work we modeled the emergence of a galactic bar. The idea was to create a system that started as a barless 
galaxy and that would become a barred galaxy later on, where the bar grew over time. In order to model this evolution of the bar potential, a linear function of time was assumed for the mass of the bar in the Ferrers potential (this was done in an external routine of the LP-VIcode, the same routine where the user provides the potential and the motion and variational equations).

With these two actions we were able to use the LP- VIcode to study the dynamics of a barred galaxy, with the mass of the bar growing over time, and remaining constantly rotating around the $z$-axis.

\section{DEVELOPMENT AND DISCUSSION}

\subsection{Models and Parameters}

We studied three models $\mathrm{X}, \mathrm{Y}$ and $\mathrm{Z}$ whose parameters are shown in Table 1. Although it is not necessary to understand the origin of these parameters for our study, the reader can find a brief description of the procedures adopted in Appendix A, where we explain that the inspiration for these parameters came from the galaxy NGC 936, and where we describe how we computed the parameters based on the works of Kent \& Glaudell (1989) and Merrifield \& Kuijken (1995). Each of the models was divided into two more specific models, where the rate of bar growth was varied (in one of them, the bar totally evolves with 5 turns around itself and in the other with 10 turns) generating in total six models: $X_{5}, X_{10}, Y_{5}, Y_{10}, Z_{5}$ and $Z_{10}$.

In Table 1 , and all along this paper, the model system of units was defined considering the gravitational constant $G=1$. We adopted $1 \mathrm{kpc}$ for length, $10^{3} \mathrm{~km} \mathrm{~s}^{-1}$ for velocity, $10^{3} \mathrm{~km} \mathrm{~s}^{-1} \mathrm{kpc}^{-1}$ for pattern speed, $1 \mathrm{Myr}$ for time, and $2 \times 10^{11} M_{\odot}$ for mass. The total mass $G\left(M_{S}+M_{D}+M_{B}\right)$ was always equal to 1 . For the energy, the unit is $10^{6} \mathrm{~km}^{2} \mathrm{~s}^{-2}$. The integration time was $10000 \mathrm{Myr}$.

For all models (X, Y and $\mathrm{Z}$ ) the masses of the bulge, disk and bar components, as well as their other parameters do not change. Therefore, the difference between these three models is basically the galactic bar pattern speed; Model X has a slower $\Omega_{b}$, model $\mathrm{Y}$ is intermediate and model $\mathrm{Z}$ has a faster $\Omega_{b}$.

The formation of a bar is a secular process that can have several histories. Bars can form, change (increase, decrease, rotate etc.) and extinguish themselves with time, in processes that depend on the parameters of the galaxies that host such bars Bournaud \& Combes (2002). No analytical studies on gravitational bar potentials that evolve over time are known. In some recent works, such as like Manos \& Machado (2014), Machado \& Manos (2016) and Chaves-Velasquez et al. (2017), the authors wrote about the barred galaxy stability using time-dependent potentials. However, these studies were based on N-body simulations of barred galaxies by extracting parameters of the simulation for certain times in the system evolution, and treating each snapshot as a time-independent model.

Therefore, our intention is to carry out a study where the gravitational potential that represents the bar evolves over time in an analytical way. The idea is that our system starts as a barless galaxy and becomes a barred galaxy, whose bar grows over time.

We began the integrations with totally axisymmetric potentials, without bar, and over time we transformed these potentials into non-axisymmetric ones, with a bar.

Let us recall that the effective potential is given by $\Phi_{e f f}(\mathbf{x})=\Phi(\mathbf{x})-\frac{1}{2}|\Omega \times \mathbf{x}|^{2}$ and the Lagrange points are five points where $\nabla \Phi_{\text {eff }}=0$. Writing the potential like this, we have a rotating system representation. The quantity $E_{J}=\frac{1}{2}|\mathbf{v}|^{2}+\Phi_{\text {eff }}$ is called the Jacobi energy and is conserved in the rotating system (for more details see Binney \& Tremaine 2008). Figure 1 shows the initial effective potentials and the final effective potentials for Models X, Y, and $\mathrm{Z}$, where the emergence of the bar can be clearly seen.

In order to perform this bar evolution (shown in Figure 1), it was decided to implement a linear timedependent function of the mass of the bar in the Ferrers potential. In this process, two specific cases were created for each model: the evolution is completed in a time corresponding to 5 or 10 complete turns of the bar around itself. With this approach, we created the $X_{5}, X_{10}, Y_{5}, Y_{10}, Z_{5}$ and $Z_{10}$ notations. The bar evolution time for each model obviously depends on the pattern speed of the bar $\Omega_{b}$ and on the length of the bar. These times were calculated to serve as parameters of the growth function of the bar and are displayed in Table 2.

The Jacobi energy $E_{J}=\frac{1}{2}|\mathbf{v}|^{2}+\Phi_{\text {eff }}(\mathbf{x})$ is conserved in a rotating potential system representative of a fixed bar. However, this energy is not conserved during the evolution. While the mass of the bar is growing linearly, the $E_{J}$ value of any particle also decreases linearly, and it is conserved again as soon as the bar growth finishes and the system bears a fixed bar (after evolution). To exemplify this statement, a random orbit was used, with an initially circular motion, integrated in Model $X_{5}$. The integration 
TABLE 1

PARAMETER SETS

\begin{tabular}{lllllllllll}
\hline & $M_{S}$ & $\epsilon_{S}$ & $M_{D}$ & $\mathrm{~A}$ & $\mathrm{~B}$ & $M_{B}$ & $\mathrm{a}$ & $\mathrm{b}$ & $\mathrm{c}$ & $\Omega_{b}$ \\
\hline Model X & 0.1273 & 0.45 & 0.7406 & 4.7 & 0.4 & 0.1321 & 4.0 & 1.1 & 0.4 & 0.05 \\
Model Y & 0.1273 & 0.45 & 0.7406 & 4.7 & 0.4 & 0.1321 & 4.0 & 1.1 & 0.4 & 0.06 \\
Model Z & 0.1273 & 0.45 & 0.7406 & 4.7 & 0.4 & 0.1321 & 4.0 & 1.1 & 0.4 & 0.07 \\
\hline
\end{tabular}

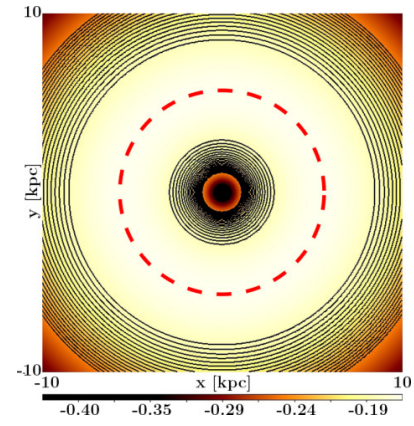

(a) Initial Model X

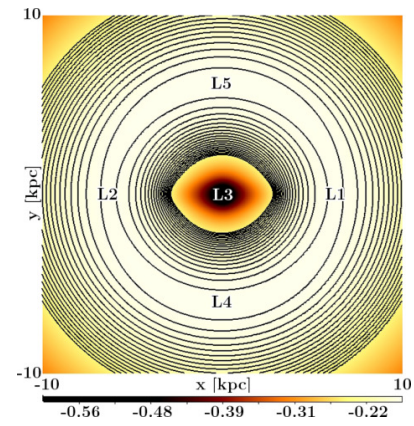

(d) Final Model X

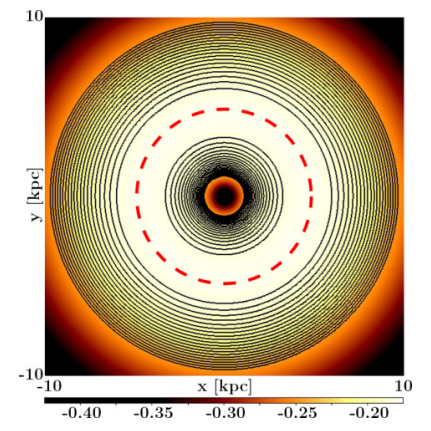

(b) Initial Model Y

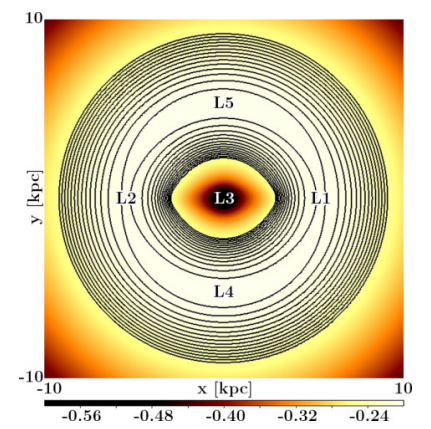

(e) Final Model Y

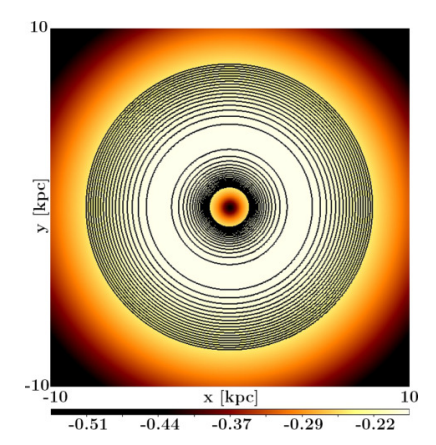

(c) Initial Model Z

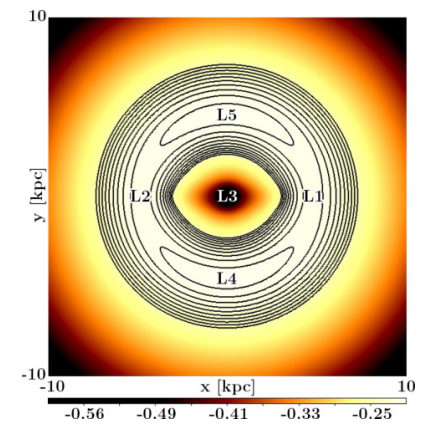

(f) Final Model Z

Fig. 1. Effective potential contours of Models X, Y and Z. The top three images illustrate the initial effective potential contours, when the models did not yet have a bar, so the potential is axisymmetric. The three bottom images illustrate the final effective potential, when the bar is fully grown. Although there is no bar formed yet in the models of the first row, the effective potential, in a coordinate system that rotates with the pattern speed of the forthcoming bar, defines a radius of corrotation which is displayed in red. All images in the bottom row display the $L 1-L 5$ Lagrange points. Twenty contours between energies -0.25 and -0.18 are displayed for each model. The color figure can be viewed online.

TABLE 2

TIME FOR BAR EVOLUTION IN EACH MODEL

\begin{tabular}{lc}
\hline Model & Time $(\mathrm{Myr})$ \\
\hline$X_{5}$ & 614.35 \\
$X_{10}$ & 1228.70 \\
$Y_{5}$ & 511.96 \\
$Y_{10}$ & 1023.92 \\
$Z_{5}$ & 438.82 \\
$Z_{10}$ & 877.65 \\
\hline
\end{tabular}

begins without the bar, the bar structure starts to emerge and its mass increases linearly until the time 614.35 Myr (as shown in Table 2). After this evo- lution, the system has a fixed bar until the end of the integration at 10,000 Myr. This whole process is displayed in Figure 2.

\subsection{Initial Conditions}

Galactic bars behave like rigid bodies, that is, $\Omega_{b}$ is always constant. However, galactic disks do not behave this way, their pattern speed $\Omega$ is a function of the radial coordinate. Thus, it is natural to imagine that resonances will appear between the bar and disk. An example is the corrotation resonance $(\mathrm{CR})$, which occurs where $\Omega=\Omega_{b}$. 


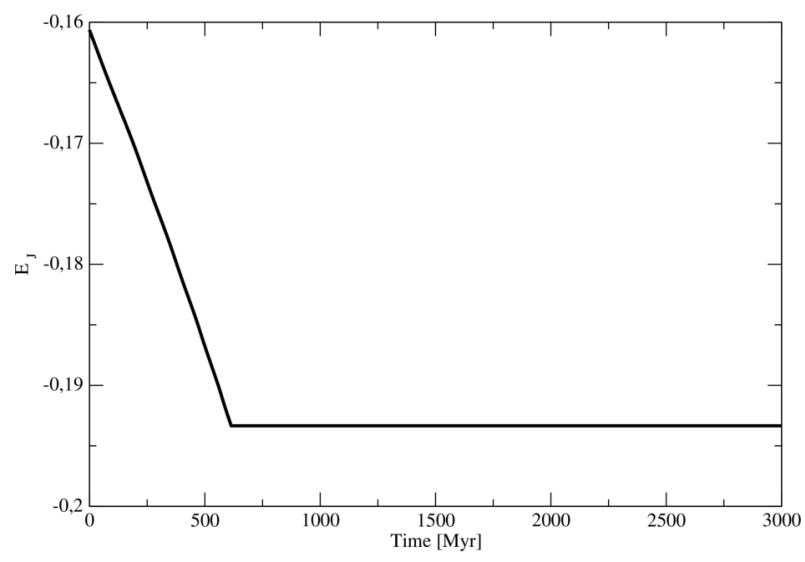

Fig. 2. This image displays the $E_{J}$ behavior for a random orbit as the bar grows in Model $X_{5}$. It can be observed that $E_{J}$ is not conserved during bar growth, but it is conserved after the bar has evolved. Notice that the evolution time is completely in agreement with that shown in Table 2 for Model $X_{5}(614.35 \mathrm{Myr})$. The integrations were made up to $10,000 \mathrm{Myr}$, and for this illustration we plot the time until 3,000 Myr. The $E_{J}$ behavior for this orbit is not unique: for all integrated orbits this energy decrease occurs during the time of bar evolution.

There will also be resonances when the following condition is satisfied:

$$
\Omega=\Omega_{b} \pm \frac{\kappa}{m},
$$

where $m$ is an integer related to the symmetry of the structure in which we are interested $(\mathrm{m}$-armed spiral structures, bars etc.), and $\kappa^{2}=\frac{d^{2} \Phi}{d R^{2}}+\frac{3}{R} \frac{d \Phi}{d R}$ is the epicyclic frequency. In this case, there will be two resonances, the Lindblad resonances. In Equation (10), for the negative sign, there is the Inner Lindblad Resonance (ILR); for the positive sign, the Outer Lindblad Resonance (OLR). For a galactic bar potential we have $m=2$ because of the bisymmetric structure. Figure 3 displays the curves $\Omega, \Omega+\frac{1}{2} \kappa$ and $\Omega-\frac{1}{2} \kappa$ for Models X, Y and Z.

As the galactic bar is expected to always be contained in the $\mathrm{CR}$ radius, its influence does not exceed the OLR radius. Therefore, we only consider orbits with initial positions inside the OLR radius. For this study, we launched particles in initially circular orbits, distributed randomly from the center up to the OLR resonance, with 10,000 prograde orbits and 10,000 retrograde orbits for each model, starting from the positions shown in Figure 4. By prograde and retrograde orbits we mean orbits launched in the direct and opposite directions, respectively, in the bar corrotating non inertial reference frame. It is important to stress that we are dealing with motions

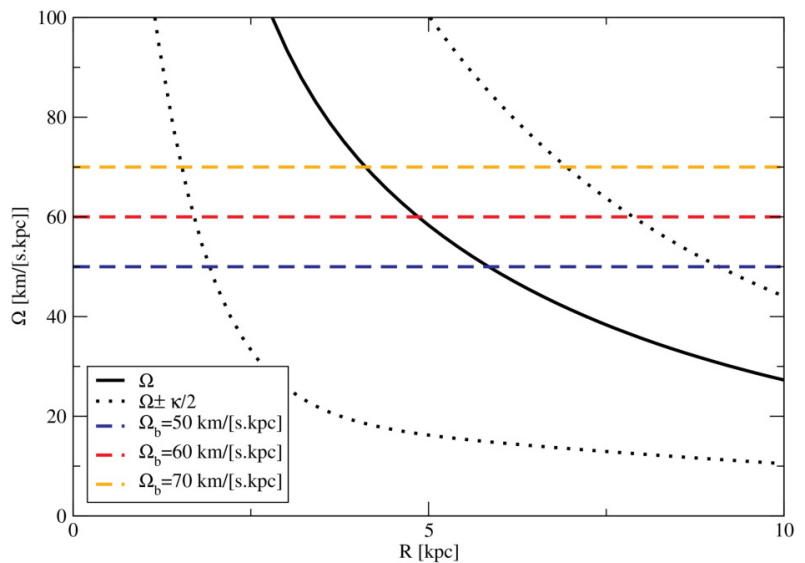

Fig. 3. $\Omega, \Omega+\frac{1}{2} \kappa$ and $\Omega-\frac{1}{2} \kappa$ curves for Models X, Y e $\mathrm{Z}$ and the corresponding CR, ILR and OLR resonances. The color figure can be viewed online.

of individual particles in a gravitational potential, i.e., this is not a self-consistent N-body simulation. The same number of prograde and retrograde orbits does not mean that we are weighting them equally, since it is known that prograde orbits play a much more important role in a barred galaxy potential. It just means we are exploring possible prograde and retrograde orbits with different initial conditions.

Notice that the greater the bar pattern speed $\Omega_{b}$, the smaller will be the OLR radius. According to our criterion for the choice of initial conditions, Model X has more scattered orbits than Model Y and, in turn, Model Y has more scattered orbits than Model Z. This phenomenon is clearly shown in Figure 4.

\subsection{Results and Discussion}

For efficiency, we inserted a condition in the $S A L I$ calculation on the LP- VIcode program to show us the moment when $S A L I<10^{-8}$, which we consider close enough to zero to classify the orbit as chaotic. With this, we were able to create a classification for the chaos level of an orbit. The categories are as follows:

Level 1: $S A L I<10^{-8}$ for $t \in[0,2500)$.

Level 2: $S A L I<10^{-8}$ for $t \in[2500,5000)$.

Level 3: $S A L I<10^{-8}$ for $t \in[5000,7500)$.

Level 4: $S A L I<10^{-8}$ for $t \in[7500,10000]$, where $t$ is measured in Myr ${ }^{5}$.

\footnotetext{
${ }^{5}$ All orbits were integrated to $10,000 \mathrm{Myr}$.
} 


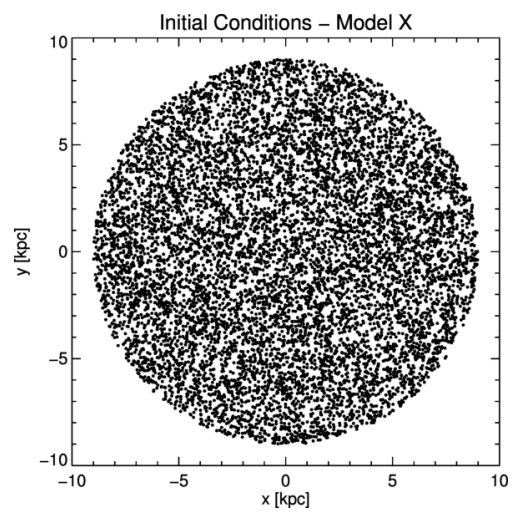

(a) Initial positions - Model X

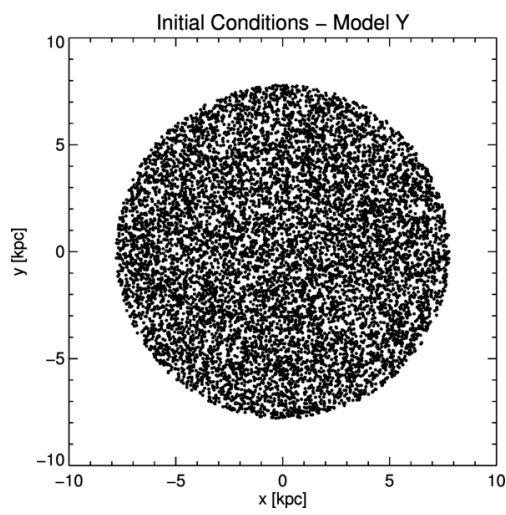

(b) Initial positions - Model Y

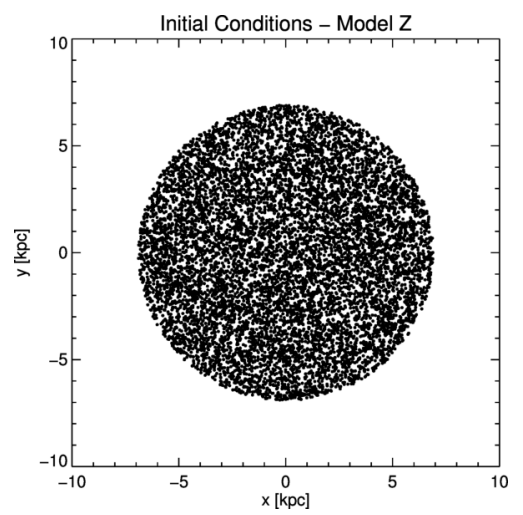

(c) Initial positions - Model Z

Fig. 4. Initial particle distributions for Models X, Y and Z. All orbits were launched with initial circular velocity and were distributed randomly inside the OLR resonance for each model. It is clear in the images that Model $\mathrm{X}$ has more scattered orbits than Model Y and, in turn, Model Y has more scattered orbits than Model Z; this happens because the greater the bar pattern speed $\Omega_{b}$, the smaller becomes the OLR radius. This image shows dots representing 10,000 initial positions. Indeed, 10,000 prograde orbits and 10,000 retrograde orbits were computed for each model, starting from these same positions.

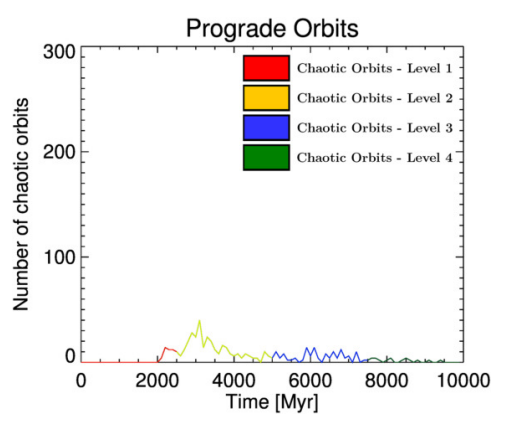

(a) Progrades - Model $X_{5}$

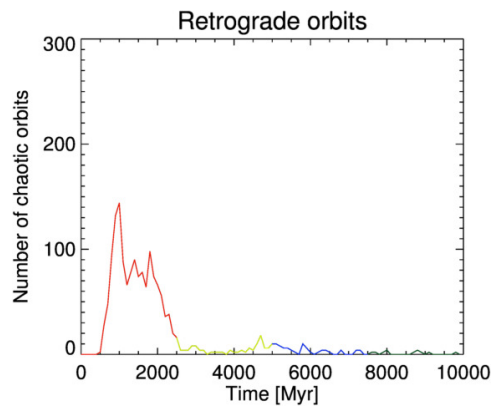

(b) Retrogrades - Model $X_{5}$

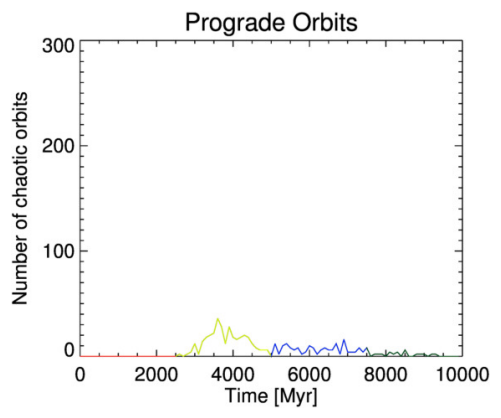

(c) Progrades - Model $X_{10}$

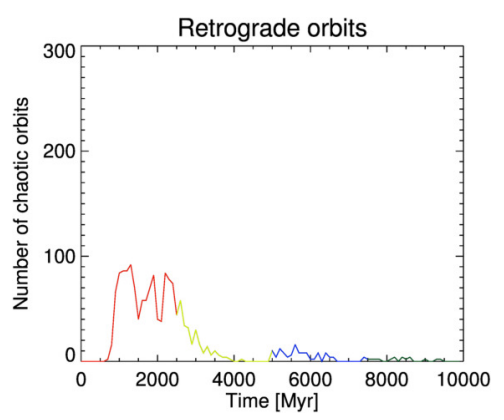

(d) Retrogrades - Model $X_{10}$

Fig. 5. Integration time $\times$ chaotic orbit number for Models $X_{5}$ and $X_{10}$. Model $X_{5}$, in which the bar grows faster, has slightly more chaos (mainly Level 1 ) than Model $X_{10}$. The colors of the line segments are defined in the legend inside the upper plot. The color figure can be viewed online. 


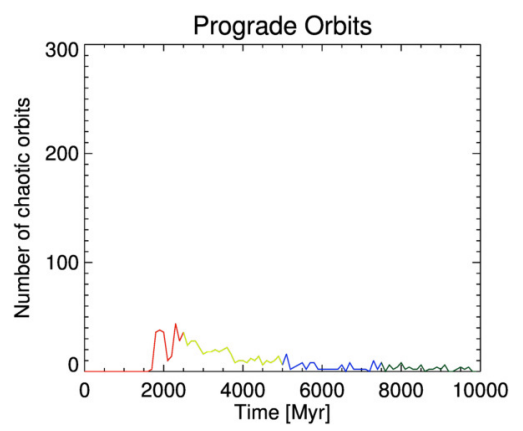

(a) Progrades - Model $Y_{5}$

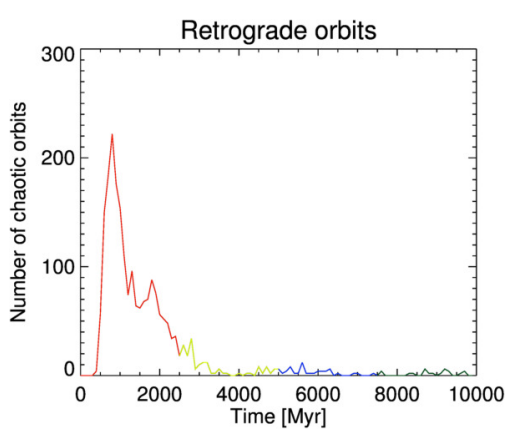

(b) Retrogrades - Model $Y_{5}$

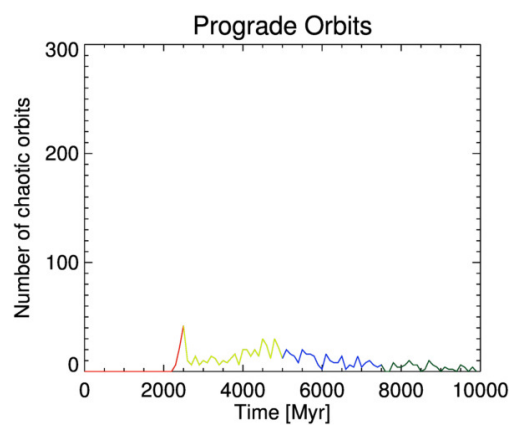

(c) Progrades - Model $Y_{10}$

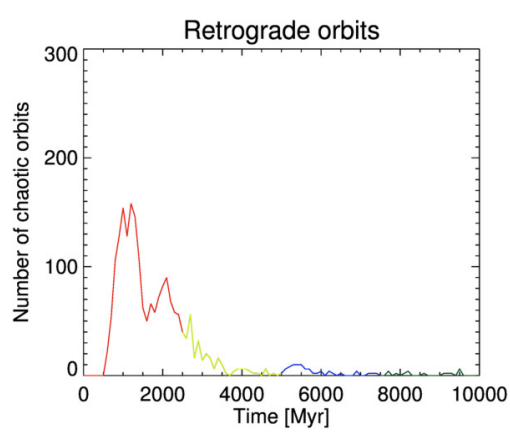

(d) Retrogrades - Model $Y_{10}$

Fig. 6. Integration time $\times$ chaotic orbit number for Models $Y_{5}$ and $Y_{10}$. Model $Y_{5}$, in which the bar grows faster, has slightly more chaos (mainly Level 1 ) than Model $Y_{10}$. The colors of the line segments are defined as in Figure 5. The color figure can be viewed online.

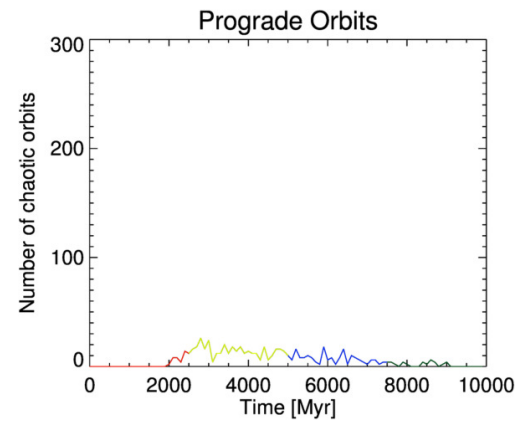

(a) Progrades - Model $Z_{5}$

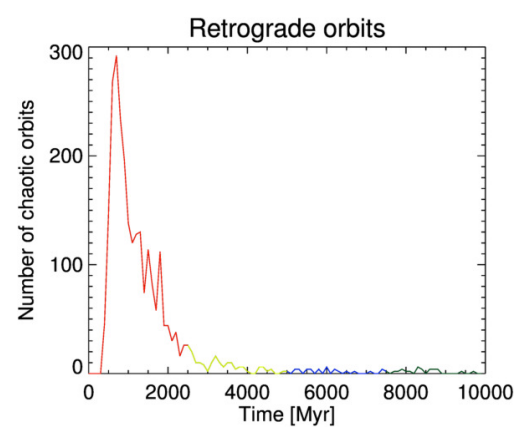

(b) Retrogrades - Model $Z_{5}$

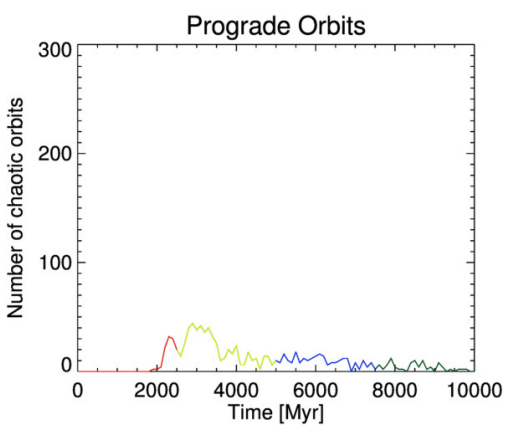

(c) Progrades - Model $Z_{10}$

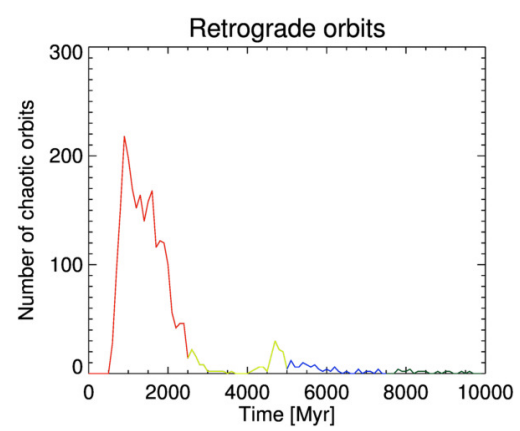

(d) Retrogrades - Model $Z_{10}$

Fig. 7. Integration time $\times$ chaotic orbit number for Models $Z_{5}$ and $Z_{10}$. Model $Z_{5}$, in which the bar grows faster, has slightly more chaos (mainly Level 1 ) than Model $Z_{10}$. The colors of the line segments are defined as in Figure 5. The color figure can be viewed online. 


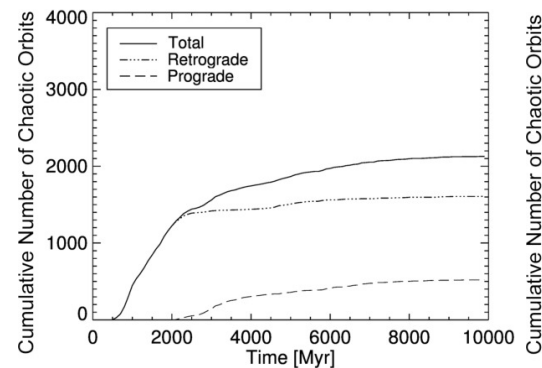

(a) Model $X_{5}$

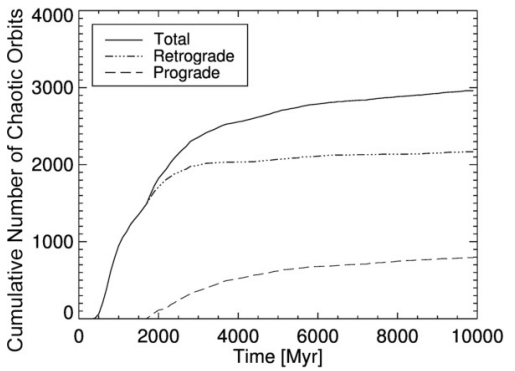

(c) Model $Y_{5}$

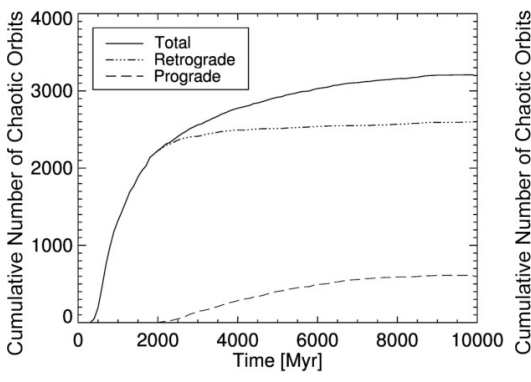

(e) Model $Z_{5}$

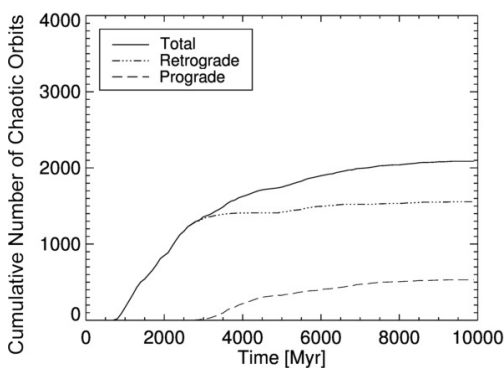

(b) Model $X_{10}$

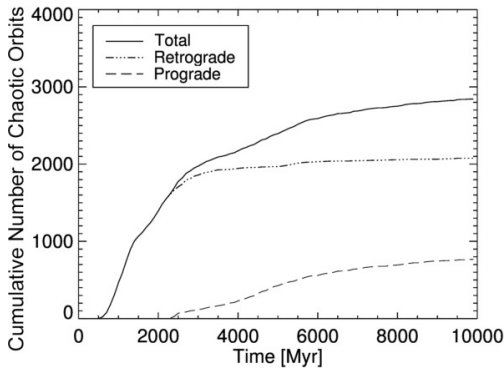

(d) Model $Y_{10}$

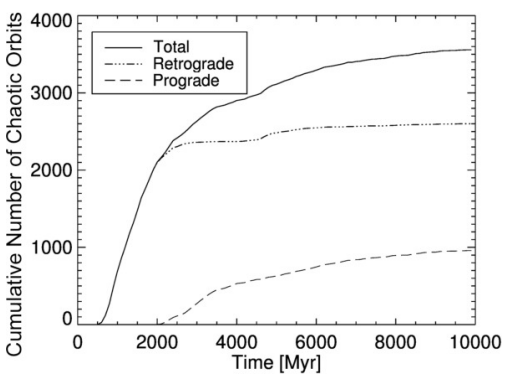

(f) Model $Z_{10}$

Fig. 8. Time $\times$ cumulative number of chaotic orbits for Models $X_{5}, X_{10}, Y_{5}, Y_{10}, Z_{5}$ and $Z_{10}$. Looking at the sequence of images, one realizes that the number of orbits that become chaotic at some point is closely related to the bar pattern speed. Clearly in Model Z, in which the bar rotates faster, there is a greater amount of chaotic motions when compared to Model X, in which the bar is slower. While Figures 5 to 7 show a slight difference indicating that models in which the bar grows faster have slightly more Level 1 chaos, here by analyzing the general context, for the cumulative number of chaotic orbits nothing can be said.

Figures 5 to 7 show the amount of chaos that arose at each integration time for each model, for both prograde and retrograde orbits. The levels of chaoticity are represented by different colors. Apparently, orbits launched as retrogrades are more conducive to chaos. This observation is consistent with Caritá et al. (2017); however it contradicts results of some classic investigations, (Athanassoula et al. 1983; and Pfenniger 1984), where there is more order in the retrograde parts of the surfaces of section of these fixed potentials. Certainly, this will lead us to further investigations in a future article.

All models studied presented strong dominant Level 1 chaos for the retrograde orbits and an appar- ent domain of Level 2 chaos for the prograde orbits. Some Level 1 chaos is generated in the prograde orbits, and specifically in Models $X_{10}$ and $Z_{5}$ this type of chaos is null or practically negligible.

Figure 8 shows the cumulative number of chaotic orbits for prograde and retrograde orbits, and for the total number of orbits of the two types in all models. The distributions between order and chaos, considering the initial positions for each model, are shown in Figures 9 to 11.

In general, for the prograde orbits, the number of chaotic orbits generated was quite low, ranging from $5 \%$ to $10 \%$ of the total number of prograde orbits launched (depending on the model analyzed). 

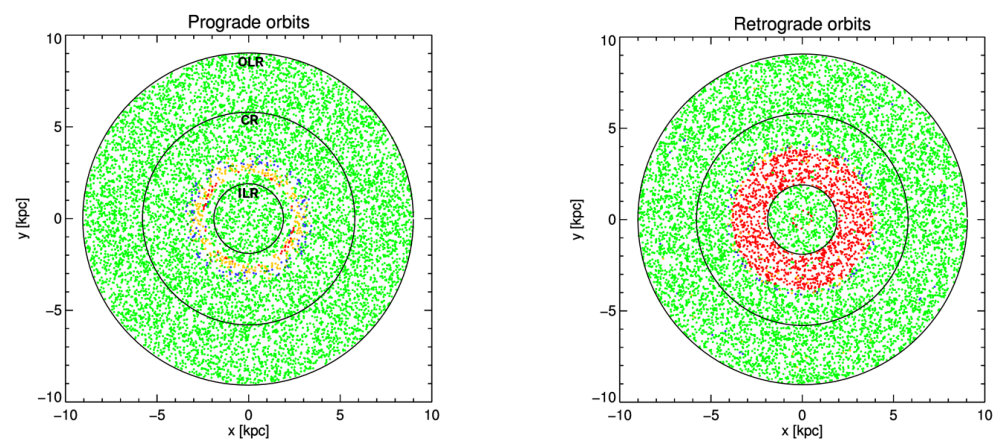

(a) Model $X_{5}$
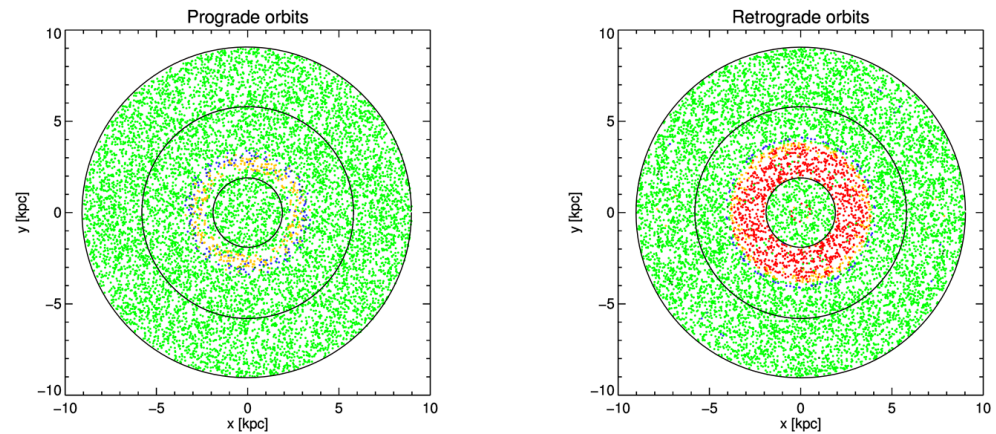

(b) Model $X_{10}$

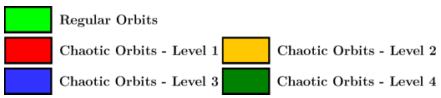

Fig. 9. Each plot shows the initial positions of all Model X particles, color-coded according to their chaos level, as in the legend above. This image again reinforces the result that orbits launched as retrogrades are more conductive to chaos. The outer limit for the inicial positions is the OLR, as stated in $\S 3.2$. The initial positions of the orbits that have become chaotic are located in a well-defined ring-like region, confined between the ILR and CR resonances. ILR, $\mathrm{CR}$ and OLR circles are shown in black, and identified in the upper left plot. Almost no orbit presents chaos with initial conditions outside these regions. The color figure can be viewed online.

On the other hand, for the retrograde orbits, this percentage increased considerably, to between $15 \%$ and $25 \%$ of the total number of retrograde orbits launched (depending on the model). In total numbers, considering the prograde and retrograde orbits, the percentage of chaos in the integrated orbits was always between $10 \%$ and $18 \%$ (depending on the model).

Figure 8 does not show appreciable changes with the rate of bar growth. However, in Figures 5 to 7 dissimilarities appear. The models where the bar grows over 5 turns seem to provide slightly more chaos than the models where the bar grows over 10 turns. This is an indication that an abrupt appearance of the bar causes more disturbance in the system.

Figures 9 through 11 show the stability of the orbits according to the initial positions. They all present a common feature: very well-defined ring- like regions of chaos. For the prograde orbits, there is only one ring of chaos for each model. For the retrograde orbits, there are two rings of chaos, a large and thick outer ring, surrounding a subtle inner ring, with one exception for Model $Z_{5}$. The CR resonance limits these rings; in this context, interestingly, the most prominent rings are confined between the ILR and CR resonances, with a single exception for Model $Z_{5}$. Very few orbits presented chaos with initial conditions outside these ring-like regions. In Figures 9 through 11 the difference in the amount of chaos for prograde and retrograde orbits is also clearly shown.

The greater the bar pattern speed $\Omega_{b}$, the smaller the OLR radius. Figures 5 to 11 also show that the greater $\Omega_{b}$, the more chaos the orbits that are within the OLR radius will present. In fact, Figures 5 through 7 make it clear that the main difference in this respect is especially in the retrograde 

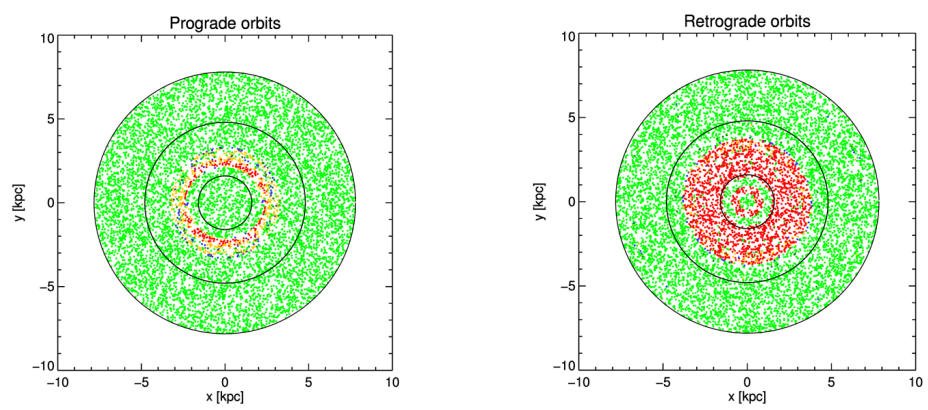

(a) Model $Y_{5}$
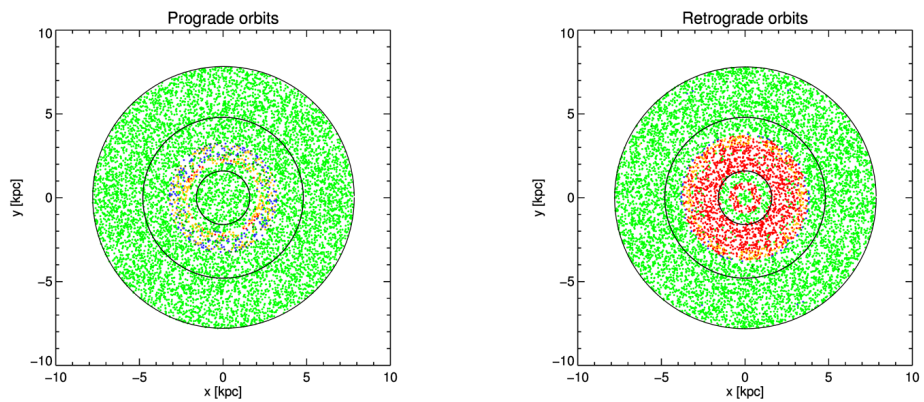

(b) Model $Y_{10}$

Fig. 10. Each plot shows the initial positions of all Model Y particles. All plots are organized and color-coded as in Figure 9. The color figure can be viewed online.
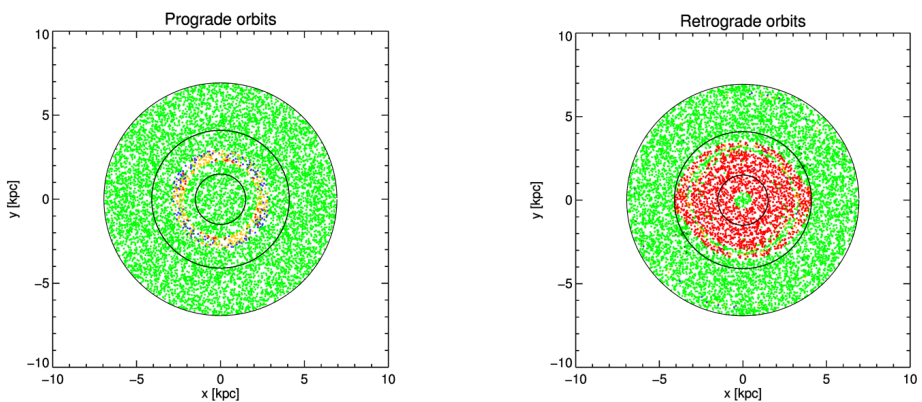

(a) Model $Z_{5}$
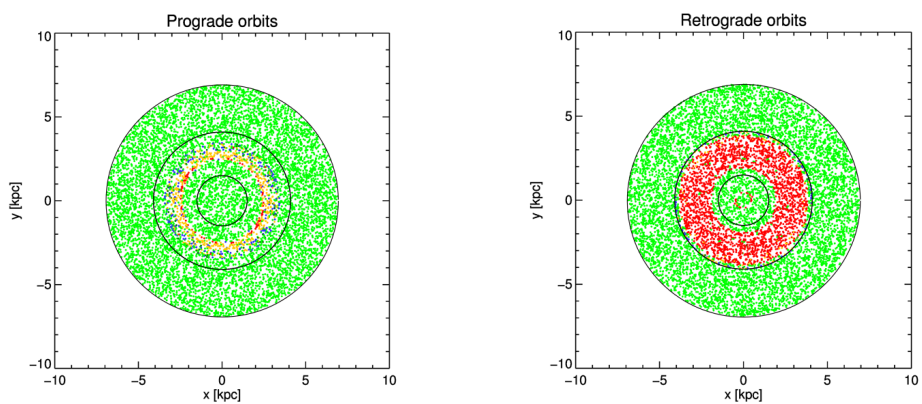

(b) Model $Z_{10}$

Fig. 11. Each plot shows the initial positions of all Model Z particles. All plots are organized and color-coded as in Figure 9. The color figure can be viewed online. 
orbits in the Level 1 of chaos. This indicates that the bar pattern speed also influences the system sensitivity to the bar appearance, since the orbits with Level 1 of chaos presented chaos in a time close to the appearance of the bar. On the other hand, for the prograde orbits, no significant differences are displayed at this point.

As expected, the $E_{J}$ of all particles is not conserved during bar evolution. As already mentioned, while the mass of the bar is growing linearly, the value $E_{J}$ also decreases linearly, and it is conserved again from the moment the bar growth finishes and the system becomes fixed (after evolution). To visualize this phenomenon, Appendix B presents some images where the number of orbits for certain times during and after the growth of the bar for each model is arranged by $E_{J}$. In these images, changes in the $E_{J}$ values can be seen until the times listed in Table 2 are reached. Afterwards, $E_{J}$ is conserved.

\section{CONCLUSIONS}

The main purpose of this work was to verify the influence of the bar on the stability of orbits in the analytical gravitational potential of barred galaxies where the bar grows over time. Six models with parameters based on observational properties of the galaxy NGC 936 were studied, and their influences on the stability of the orbits were compared. The bar dimensions were maintained in all six models and the difference between these six models was the bar pattern speed and the time of growth.

We find evidence that when the bar grows faster, more chaos is generated. For the prograde orbits, the number of chaotic orbits generated was quite low, ranging from $5 \%$ to $10 \%$ of the total number of prograde orbits launched (depending on the model). For the retrograde orbits, this percentage increased considerably, to between $15 \%$ and $25 \%$ of the total number of retrograde orbits launched (depending on the model). In this context, retrograde orbits were more conducive to chaos. This last statement provides an opportunity for further investigation, which we will conduct in the future, as it apparently contradicts some classic results (Athanassoula et al. 1983; Pfenniger 1984). We found, as expected, that $E_{J}$ was not conserved while the bar was evolving but it started to be conserved when the system stabilized. We also noted that the higher $\Omega_{b}$, the greater its influence on the orbital dynamics.

Well-defined ring-like regions of chaos were found corresponding to different initial positions, with few orbits presenting chaos outside these regions. For the prograde orbits, there was an unique ring for each model. For the retrograde orbits, two rings of chaos appeared, almost always a large, thick outer ring, surrounding a subtle inner ring. The $\mathrm{CR}$ radius was the outer limit for these chaos rings, and the most prominent rings were predominantly confined between the ILR and CR resonances.

We analyzed consistent barred galaxy models for systems in rotation and studied the orbit stability using the SALI Method. The LP-VIcode program met all of our needs and only small adjustments were needed.

We acknowledge the Brazilian agencies $\mathrm{CNPq}$ (200906-2015-1), CAPES and FAPESP, as well as the Mexican agency CONACyT (CB-2014-240426) for supporting this work. Our sincere thanks to Dr. Pfenniger, who kindly provided us with his Fortran 77 implementation of the Ferrers bar potential. All numerical work was developed using the Hipercubo Cluster resources (FINEP 01.10.0661-00, FAPESP 2011/13250-0 and FAPESP 2013/17247-9) at IP\&DUNIVAP.

\section{APPENDIX}

\section{A. CHOICE OF PARAMETERS - NGC 936}

NGC 936 is a barred spiral galaxy, type SB0 in the Hubble scheme (Hubble 1926), which is about 19.6 Mpc away in the direction of Cetus. This galaxy has a very prominent bar and bulge, and a ring structure that surrounds the bar. It was discovered on January 6,1785 by William Herschel and was classified at the time as a planetary nebula, because of its round shape (Herschel 1785a,b).

As the models described in the paper (Plummer, Miyamoto-Nagai and Ferrers) had already been implemented and were working well in LP-VIcode (Caritá et al. 2017), we decided to maintain the same modeling for this research with bars that grow over time. In order to adjust the necessary parameters for NGC 936, the works of Kent \& Glaudell (1989) and Merrifield \& Kuijken (1995) were used. We note that the Plummer, Miyamoto-Nagai and Ferrers models may not be the best for modeling galaxy NGC 936; but we emphasize that this galaxy was used only as an inspiration for our parameters. The procedures for finding the parameters are described below.

Kent \& Glaudell (1989) proposed an analytical model of the NGC 936 bulge given by a truncated King model (King 1962). The model density is:

$$
\rho(s)=\rho_{c}\left(\frac{1}{\left[1+\left(\frac{s}{a}\right)^{2}\right]^{\frac{3}{2}}}-\frac{1}{\left[1+\left(\frac{s_{c}}{a}\right)^{2}\right]^{\frac{3}{2}}}\right),
$$


where $\rho_{c}=22 L_{\odot} \mathrm{pc}^{-3}, a=265 \mathrm{pc}, s_{c}=$ $2.7 \mathrm{kpc}$ and the radial coordinate $s$ is given by $s^{2}=x^{2}+y^{2}+(z / 0,63)^{2}$.

By varying the parameters $\epsilon_{S}$ and $M_{S}$ in the Plummer Model, calculating the cumulative mass curve for this model and comparing it with the King model cumulative mass curve, using the smallest variance between the corresponding points in the graphs, we could estimate parameters $\epsilon_{S}$ and $M_{S}$ for a better fit. The fit of these curves was done up to the radius $2.7 \mathrm{kpc}$ and, thus, we were able to estimate the values $\epsilon_{S}=0.45$ and $M_{S}=5.4 \times 10^{9} M_{\odot}$.

The work of Kent \& Glaudell (1989) also allowed us to extract an approximation for the brightness profile for the disk together with the bar, as follows:

$$
\Sigma=\Sigma_{0} e^{-r / h}
$$

where $\Sigma_{0}=355 L_{\odot} \mathrm{pc}^{-2}$ and $h=3.5 \mathrm{kpc}$.

By adjusting the mass growth curves and using the smallest variance (as we did with the bulge), we were able to estimate the Miyamoto-Nagai parameters $A$ and $B$, as well as the mass of the disk plus the mass of the bar. The fit of the curves was done up to a radius of $10 \mathrm{kpc}$ and, thus, we were able to estimate the values $A=4.7, B=0.4$ and $M_{\text {disc }+ \text { bar }}=3.7 \times 10^{10} M_{\odot}$.

For the bar, parameters $a=4 \mathrm{kpc}$ and $b=$ $1.1 \mathrm{kpc}$ of the Ferrers potential were extracted from Kent \& Glaudell (1989), with dimensions $8.0 \times 2.2 \mathrm{kpc}$. From this same work, the mass of the bar was extracted, using the luminosity information as $5.6 \times 10^{9} L_{\odot}$. From this fact, using the relation $\frac{M}{L}=1$ we extracted that the mass of the bar $M_{B}=5.6 \times 10^{9} M_{\odot}$. The parameter $c=0.4 \mathrm{kpc}$ was taken for convenience only.

According to Merrifield \& Kuijken (1995), the bar pattern speed of the galaxy NGC 936 is estimated to be $\Omega_{b}=60 \pm 14 \mathrm{~km} \mathrm{~s}^{-1} \mathrm{kpc}^{-1}$. With this, we decided to establish three models by varying the bar pattern speed. The values $\Omega$ considered by us and their respective models were chosen once the NGC 936 bar pattern speed was estimated as $\Omega_{b}=60 \pm 14 \mathrm{~km} \mathrm{~s}^{-1} \mathrm{kpc}^{-1}$ (Merrifield \& Kuijken 1995); these values are shown in Table 3.
TABLE 3

\section{MODELS X, Y AND Z VARYING THE BAR PATTERN SPEED OF NGC 936}

\begin{tabular}{lll}
\hline Model X & Model Y & Model Z \\
\hline$\Omega_{b}=50 \frac{\mathrm{km}}{\mathrm{s} \mathrm{kpc}}$ & $\Omega_{b}=60 \frac{\mathrm{km}}{\mathrm{skpc}}$ & $\Omega_{b}=70 \frac{\mathrm{km}}{\mathrm{skpc}}$ \\
\hline
\end{tabular}

Recall that the model units adopted were: $1 \mathrm{kpc}$ for length, $10^{3} \mathrm{~km} \mathrm{~s}^{-1}$ for velocity, $10^{3} \mathrm{~km} \mathrm{~s}^{-1} \mathrm{kpc}^{-1}$ for pattern speed, 1 Myr for time, and $2 \times 10^{11} M_{\odot}$ for mass. The universal gravitational constant $G$ was always equal to 1 and the total mass $G\left(M_{S}+M_{D}+M_{B}\right)$ was always equal to 1 . The integration time was $10000 \mathrm{Myr}$. The following ratios were calculated: $\frac{M_{S}}{M_{T}}=\frac{0.4 \times 10^{9}}{42.4 \times 10^{9}} \approx$ $0.1273, \frac{M_{D}}{M_{T}}=\frac{31.4 \times 10^{9}}{42.4 \times 10^{9}} \approx 0.7406$ and $\frac{M_{B}}{M_{T}}=$ $\frac{5.6 \times 10^{9}}{42.4 \times 10^{9}} \approx 0.1321$. To avoid confusion in the notations, we chose to use $M_{S}, M_{D}$ and $M_{B}$ for the ratios. In this way the models presented in Table 1 were constructed.

\section{B. $E_{J}$ BEHAVIOR}

The Jacobi Energy is not conserved during bar evolution. While the bar mass is growing linearly, the value $E_{J}$ per particle decreases linearly, and it is conserved again from the moment the bar growth finishes and the system becomes fixed (after evolution).

The behavior of the Jacobi energy is shown in Figure 2. In order to better visualize this phenomenon, the number of orbits per $E_{J}$ for certain times during and after the growth of the bar is arranged in Figure 12 for each model. In these images it is possible to observe that the value of $E_{J}$ decreases until the times shown in Table 2 , after which time $E_{J}$ remains constant. 

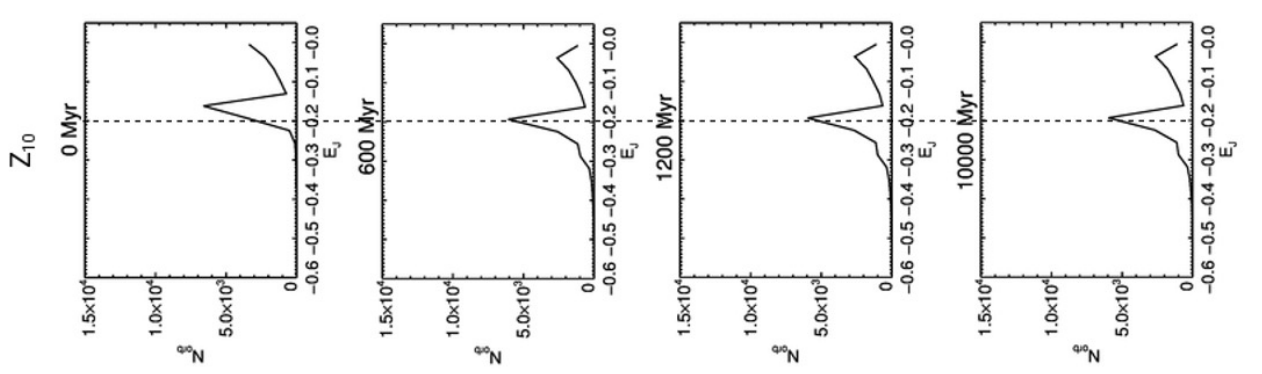

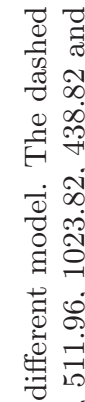
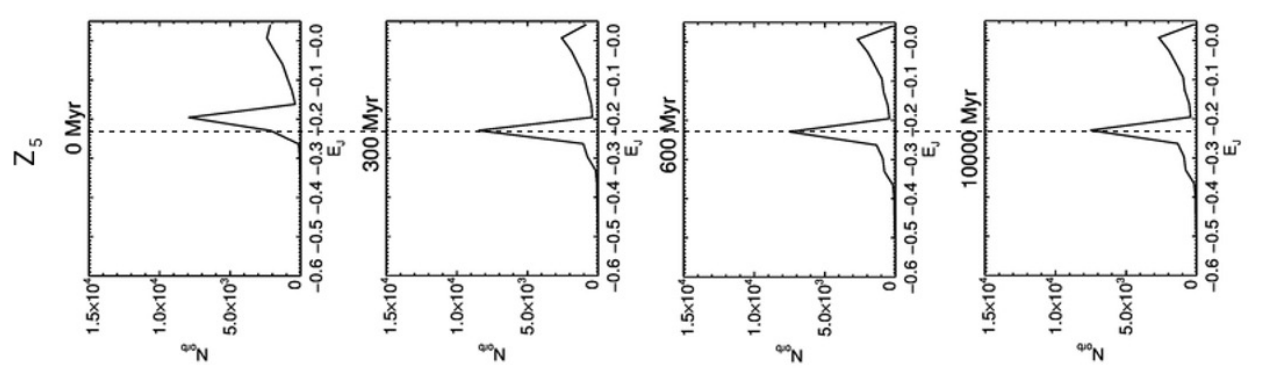

0
0
0
0
0
0
0
0
0
0
0
0
0
0
0
0
0
0
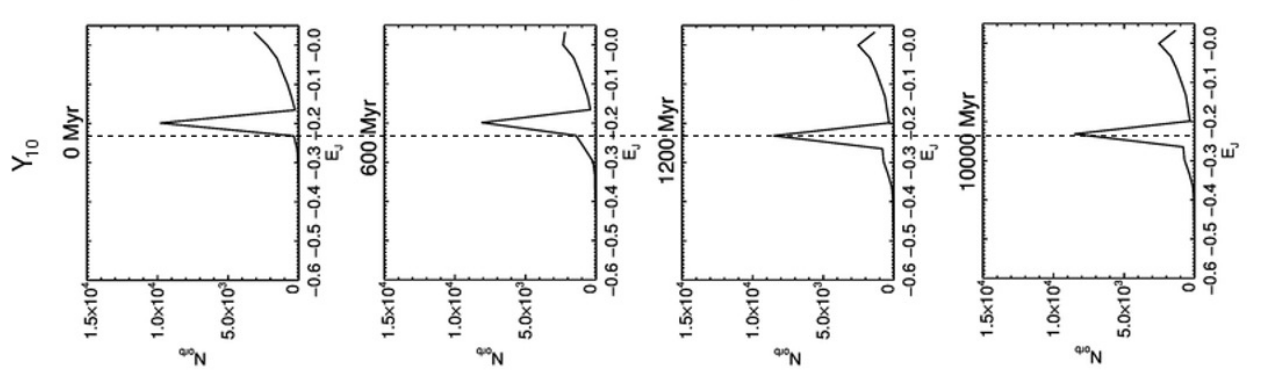

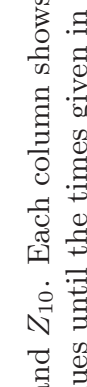
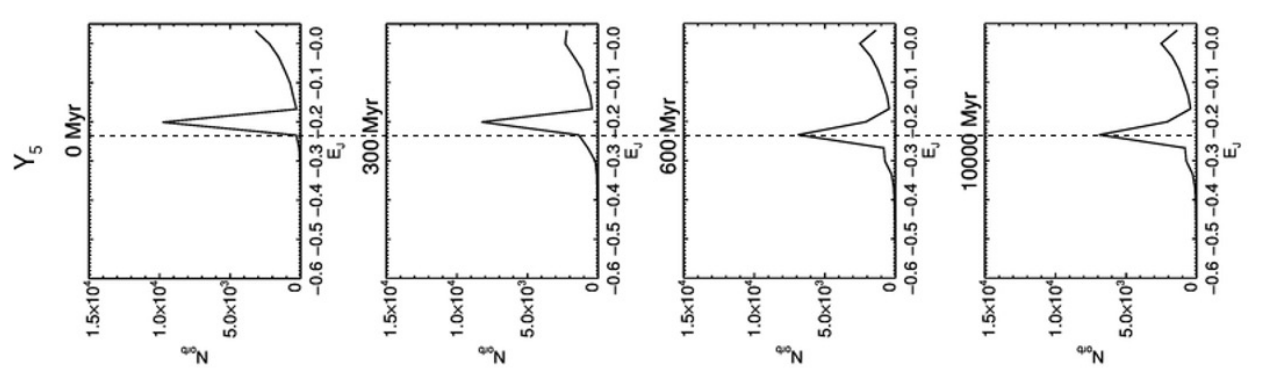

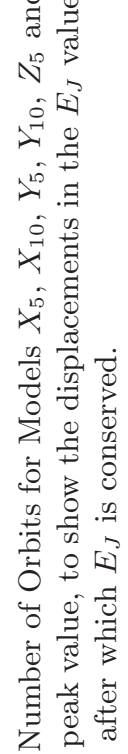
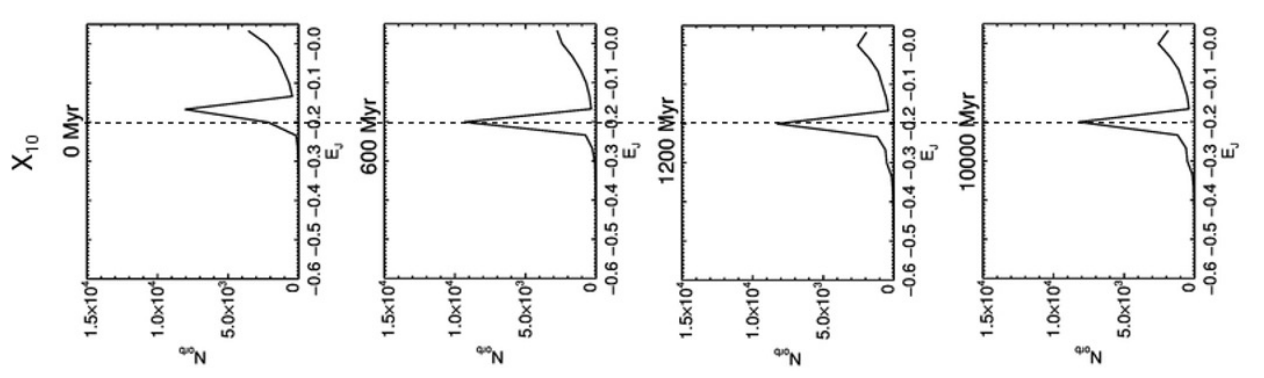

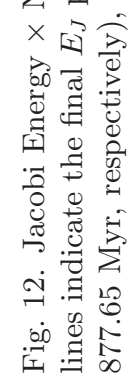

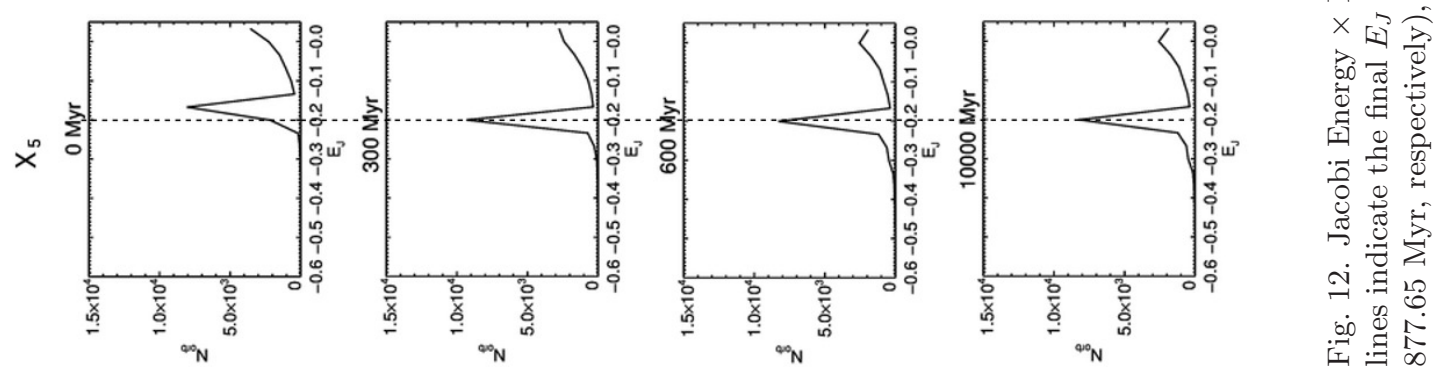




\section{REFERENCES}

Athanassoula, E., Bienayme, O., Martinet, L., \& Pfenniger, D. 1983, A\&A, 127, 349

Binney, J. \& Tremaine, S. 2008, Galactic Dynamics, (2nd ed.; Princeton, NJ: PUP)

Bournaud, F. \& Combes, F. 2002, A\&A, 392, 83

Caritá, L., Rodrigues, I., \& Puerari, I. 2017, Galax, 5, 101

Caritá, L. A., Rodrigues, I., Puerari, I., \& Schiavo, L. E. C. A. 2018, NewA, 60, 48

Carpintero, D. D., Maffione, N., \& Darriba, L. 2013, La Plata Variational Indicators code: a program to compute a suite of variational chaos indicators (User's Guide for Version 102[Kaos]) 2014, A\&C, 5, 19

Chaves-Velasquez, L., Patsis, P. A., Puerari, I., Skokos, C., \& Manos, T. 2017, ApJ, 850, 145

Eskridge, P. B., Frogel, J. A., Pogge, R. W., et al. 2000, AJ, 119, 536

Ferrers, N. M. 1877, QJPAM, 14, 1

Gajda, G., Łokas, E. L., \& Athanassoula, E. 2016, ApJ, 830, 108

Herschel, W. 1785a, RSPT, 75, 40 1785b, RSPT, 75, 213

Hubble, E. P. 1926, ApJ, 64

Kaufmann, D. E. \& Patsis, P. A. 2005, ApJ, 624, 693

Kent, S. M. \& Glaudell, G. 1989, AJ, 98, 1588
King, I. 1962, AJ, 67, 471

Machado, R. E. G. \& Manos, T. 2016, MNRAS, 458, 3578

Manos, T. \& Athanassoula, E. 2011, MNRAS, 415, 629

Manos, T. \& Machado, R. E. G. 2014, MNRAS, 438, 2201

Merrifield, M. R. \& Kuijken, K. 1995, MNRAS, 274, 933

Miyamoto, M. \& Nagai, R. 1975, PASJ, 27, 533

Patsis, P. A. 2002, Disks of Galaxies: Kinematics, Dynamics and Peturbations, ed. E. Athanassoula, A. Bosma, \& R. Mujica, ASPC, 275, 161

Patsis, P. A. \& Katsanikas, M. 2014, MNRAS, 445, 3525

Patsis, P. A., Skokos, H., \& Athanassoula, E. 2002, MNRAS, 337, 578 . 2003, MNRAS, 342, 69

Pfenniger, D. 1984, A\&A, 134, 373

Plummer, H. C. 1911, MNRAS, 71, 460

Sheth, K., Regan, M. W., Scoville, N. Z., \& Strubbe, L. E. 2003, ApJ, 592, L13

Skokos, H. 2001, JPhA, 34, 10029

Skokos, H., Antonopoulos, C., Bountis, T. C., \& Vrahatis, M. N. 2002a, eprint arXiv:nlin/0210053 . 2003, PThPS, 150, 439 2004, JPhA, 37, 6269

Skokos, H., Patsis, P. A., \& Athanassoula, E. 2002b, MNRAS, 333, 847 2002c, MNRAS, 333, 847 2002d, MNRAS, 333, 861

Lucas Antonio Caritá: Instituto Federal de Educação, Ciência e Tecnologia de São Paulo (IFSP), São José dos Campos, Brasil (prof.carita@ifsp.edu.br).

Lucas Antonio Caritá, Irapuan Rodrigues, and Luiz Eduardo Camargo Aranha Schiavo: Universidade do Vale do Paraíba (UNIVAP), São José dos Campos, Brasil.

Lucas Antonio Caritá and Ivânio Puerari: Instituto Nacional de Astrofísica, Óptica y Electrónica (INAOE), Puebla, México.

Luiz Eduardo Camargo Aranha Schiavo: Universidade Estadual de Campinas (UNICAMP), Campinas, Brasil. 